\title{
SOME RESTRICTIONS ON FINITE GROUPS ACTING FREELY ON $\left(S^{n}\right)^{k}$
}

\author{
BY \\ GUNNAR CARLSSON ${ }^{1}$
}

\begin{abstract}
Restrictions other than rank conditicns on elementary abelian subgroups are found for finite groups acting freely on $\left(S^{n}\right)^{k}$, with trivial action on homology. It is shown that elements $x$ of order $p, p$ an odd prime, with $x$ in the normalizer of an elementary abelian 2-subgroup $E$ of $G$, must act trivially on $E$ unless $p \mid(n+1)$. It is also shown that if $p=3$ or $7, x$ must act trivially, independent of $n$. This produces a large family of groups which do not act freely on $\left(S^{n}\right)^{k}$ for any values of $n$ and $k$. For certain primes $p$, using the mod two Steenrod algebra, one may show that $x$ acts trivially unless $2^{\mu(p)} \mid(n+1)$, where $\mu(p)$ is an integer depending on $p$.
\end{abstract}

Introduction. In this paper, we will describe some restrictions of the structure of finite groups $G$ which act freely on spaces having the homotopy type $\Pi_{j-1}^{k} S^{n}$. For the case of $k=1$, it is well known that all abelian subgroups of $G$ are cyclic, and this condition is sufficient to allow the construction of a finite $\mathrm{CW}$-complex on which $G$ acts freely. In [2], it was proven that for $k$ arbitrary, the elementary abelian 2-subgroups of $G$ must have rank $\leqslant k$. However, for $k \geqslant 1$, more subtle restrictions than rank conditions are necessary. The following is known.

TheOREM [5]. $A_{4}$ does not act freely on $S^{n} \times S^{n}$, with trivial action on mod 2 homology. ${ }^{2}$

In this paper we apply the results of [2] to generalize this result considerably; thus, the main theorem, Theorem 4.4 .

THEOREM. Let $G$ be a semidirect product $\mathbf{Z} / 3 \times_{T}(\mathbf{Z} / 2 \times \mathbf{Z} / 2)$ or $\mathbf{Z} / 7 \times_{T}(\mathbf{Z} / 2$ $\times \mathbf{Z} / 2 \times \mathbf{Z} / 2$ ), with nontrivial action. Then $G$ does not act freely on a finite $C W$-complex $X, X \simeq \Pi_{j=1}^{k} S^{n}$, with trivial action on mod 2 homology, for any values of $k$ and $n$.

For fixed values of $n$, we obtain stronger restrictions on $G$, in Theorem 3.5.

THEOREM. Let $G$ act freely on $X, X \simeq \Pi_{j=1}^{k} S^{n}$, where $X$ is finite, and acts trivially on $H_{*}(X ; \mathrm{Z} / 2)$. Let $x \in N_{G}(E)$ be an odd order element of order $p, p$ prime, where $N_{G}$ denotes normalizer and $E$ is an elementary abelian subgroup of $G$. Then, unless $p \mid(n+1), x$ is in the centralizer of $E$. Moreover, there is a function $\mu: \mathbf{Z} \rightarrow \mathbf{Z}$, such that $x$ is in the centralizer unless $2^{\mu(d)} \mid(n+1) .(\mu$ is nontrival, i.e. $\mu(31)=3$.)

Received by the editors September 24, 1979 and, in revised form, March 19, 1980.

1980 Mathematics Subject Classification. Primary 55G10, 57E25.

'Supported in part by NSF Grant MCS-7903192.

${ }^{2} R$. Oliver informs me that he can also prove this theorem for $\left(S^{n}\right)^{k}$. 
EXAmple. Suppose $x \in N_{G}(E)$ has order 127, and acts nontrivially on $E$. Then $16 \cdot 127 \mid(n+1)$.

These conditions superficially resemble the Milnor condition (see [4]), for $k=1$. However, the Milnor condition is a restriction on groups acting freely on a manifold $M, M \simeq S^{n}$. Our conditions depend only on the 2-local homotopy type of the space $X$. Secondly, the Milnor condition depends on $k$; the group $D_{6}$ will act freely on $S^{n} \times S^{n}$, but not on $S^{n}$. Our conditions are independent of $k$.

1. Preliminaries. Let $\Gamma$ be a finite group of odd order, and let $\rho: \Gamma \rightarrow \mathrm{GL}_{n}(\mathbf{Z} / 2)$ be an $n$-dimensional linear representation of $\Gamma$ over the field $k=Z / 2$. We define $\Sigma(\Gamma, \rho)$ by

$$
\sum(\Gamma, \rho)=\Gamma \underset{\rho}{\times}(\mathbf{Z} / 2)^{n},
$$

the semidirect product of $\Gamma$ with $(\mathbf{Z} / 2)^{n}$. Recall that the $\mathbf{Z} / 2$-cohomology of $(\mathbf{Z} / 2)^{n}$ is given by

$$
H^{*}\left((\mathrm{Z} / 2)^{n} ; \mathrm{Z} / 2\right) \simeq(\mathrm{Z} / 2)\left[x_{1}, \ldots, x_{n}\right] \stackrel{\text { (defn.) }}{=} A_{*},
$$

the polynomial ring on $n$ 1-dimensional generators $x_{1}, \ldots, x_{n}$. Note that if $\Gamma$ is a group of automorphisms of $(\mathbf{Z} / 2)^{n}$, then $\Gamma$ acts on $A_{*}$; in fact, if $M$ is the matrix describing the action of $\gamma \in \Gamma$ on $(\mathrm{Z} / 2)^{n}$, then $\gamma$ acts on $H^{1}\left((\mathrm{Z} / 2)^{n} ; \mathrm{Z} / 2\right)=k \cdot x_{1}$ $+\cdots+k \cdot x_{n}$ by the matrix $M^{t}$, and the action extends to the rest of $A_{*}$ by multiplicativity.

Proposition 1. Let $\Gamma$ be an odd order group, $\rho: \Gamma \rightarrow \mathrm{GL}_{n}(k)$ a representation as above. Then $H^{*}(\Sigma(\Gamma, \rho) ; k) \cong A_{*}^{\Gamma}$, the ring of invariants under the action of $\Gamma$ on $A_{*}$.

Proof. Consider the Hochschild-Serre spectral sequence for the group extension

$$
(\mathrm{Z} / 2)^{n} \rightarrow \sum(\Gamma, \rho) \rightarrow \Gamma .
$$

$E_{2}^{p, q}=0$ for $p \neq 0$ and $E_{2}^{0, q}=A_{q}^{\Gamma}$; hence the result.

Recall that since $H^{*}(G ; k)$ is the cohomology ring of the space $B G$ for any group $G$, it is an algebra over the mod 2 Steenrod algebra $\mathcal{Q}(2)$. In particular, $A_{*}$ is such an algebra, and the action of $\mathcal{Q}(2)$ on $A_{*}$ is determined by the Cartan formula and the requirements $\mathrm{Sq}^{1} x_{j}=x_{j}^{2}, \mathrm{Sq}^{i} x_{j}=0$ for $i>1$.

We recall the inductive definition of the Milnor primitives $Q_{i}$ by

$$
Q_{1}=\mathrm{Sq}^{1}, \quad Q_{i+1}=\left[Q_{i}, \mathrm{Sq}^{2^{i}}\right] \text {. }
$$

Conventionally, as in [1], we define a derivation $Q_{0}$ on $A_{*}$ by $Q_{0} x_{i}=x_{i}$, and the requirement that $Q_{0}$ be a derivation. We remark that under left multiplication of elements of $A_{*}$, the derivations of $A_{*}$ to itself become a graded $A_{*}$-module.

Proposition 2 (SEE [1]). (a) $Q_{i}$ is a derivation of $A_{*}$.

(b) There exist polynomials $\varphi_{l j} \in A_{*}, j=0, \ldots, n-1, l>n$, such that $Q_{l}=$ $\sum_{j=1}^{n-1} \varphi_{l j} Q_{j}$.

(c) $Q_{0}(z)=0$ for $z \in A_{2 k}, Q_{0}(z)=z$ for $z \in A_{2 k+1}$.

Proposition 3 [1]. Let $\theta \in A_{*}$ satisfy $Q_{i} \theta=0 \forall i$. Then $\theta$ is a square. 
Corollary 4. If $\theta \in A_{*}$ satisfies $Q_{j} \theta=0$ for $j=0, \ldots, n-1$, then $\theta$ is $a$ square.

Finally, we recall some results from [2]. Let $G$ denote a finite group acting freely on a finite $C W$-complex $X$, where $X \simeq \Pi_{j=1}^{k} S^{n}$ so that the action of $G$ on $H^{*}(X ; k)$ is trivial. Let $y_{j} \in H^{n}(X)$ denote the dual to the fundamental class of the $j$ th sphere. Consider the Serre spectral sequence for the fibration

$$
X \rightarrow E G \underset{G}{\times} X \rightarrow B G
$$

where $E G$ denotes a contractible space on which $G$ acts freely. Define $f_{j} \in$ $H^{n+1}(B G ; k)=H^{n+1}(G ; k)$ to be $d_{n+1}\left(y_{j}\right)$ in the above spectral sequence.

Proposition 5 [2]. The ideal in $H^{*}(G ; k)$ generated by the $f_{j}^{\prime} s$ is $\mathcal{Q}(2)$-invariant.

Let $C_{*}$ denote a graded $k$-algebra, and let $\left\{\theta_{1}, \ldots, \theta_{k}\right\} \in C_{*}$ be a collection of homogeneous elements in $C_{*}$.

DEFINITION 6. We say $\left\{\theta_{1}, \ldots, \theta_{k}\right\}$ is a homogeneous system of parameters (h.s.o.p.) for $C_{*}$ if $C_{*}$ is a finitely generated module over the subalgebra generated by the $\theta_{j}$ 's.

REMARK. This terminology is nonstandard in that the usual definition would require the set $\left\{\theta_{1}, \ldots, \theta_{k}\right\}$ to be algebraically independent.

THEOREM 7 [2]. If $G=(\mathrm{Z} / 2)^{n}$, and $X$ and the $f_{j}$ 's are as above, then the $f_{j}$ 's form an h.s.o.p. for $A_{*}=H^{*}\left((\mathrm{Z} / 2)^{n} ; k\right)$.

Corollary 8. If $G=\Sigma(\Gamma, \rho)$, and $X$ and the $f_{j}$ 's are as above, then the $f_{j}$ 's form an h.s.o.p. for $A_{*}^{\Gamma}=H^{*}(\Sigma(\Gamma, \rho) ; k)$.

Proof. $A_{*}$ is finitely generated as an $A_{*}^{\Gamma}$-module.

2. Invariant theory for odd order cyclic groups. We wish to study groups of the type $\Sigma(\mathbf{Z} / n \mathbf{Z} ; \rho)$, where $\rho$ is a faithful, irreducible representation of $\mathbf{Z} / n \mathbf{Z}$ over $k, n$ odd. The following four propositions are standard results in the representation theory of finite groups. We omit the proofs and refer the reader to [8]. We describe the faithful, irreducible representations of $\mathbf{Z} / n \mathbf{Z}$ over $k$. Let $k\left(\zeta_{n}\right)$ denote the field obtained from $k$ by adjoining the $n$th roots of unity, and let $d_{n}=\left[k\left(\zeta_{n}\right): k\right]$. Let $T_{n} \subseteq k\left(\zeta_{n}\right)$ consist of the $n$th roots, and let $G=G\left(k\left(\zeta_{n}\right) \mid k\right) \simeq \mathbf{Z} / d_{n} \mathbf{Z}$ denote the Galois group of $k\left(\zeta_{n}\right)$ over $k$. Note that $G$ acts on $T_{n}$, and consequently on $\operatorname{Hom}\left(\mathbf{Z} / n \mathbf{Z} ; T_{n}\right)$.

Proposition 1. Any injection $\varphi \in \operatorname{Hom}\left(\mathbf{Z} / n \mathbf{Z}, T_{n}\right)$ defines an irreducible, faithful representation of $\mathbf{Z} / n \mathbf{Z}$, with $k\left(\zeta_{n}\right) \cong k^{d_{n}}$ as representation space. Conversely, any irreducible, faithful representation of $\mathbf{Z} / n \mathbf{Z}$ is obtained in this way, and two injections $\varphi, \psi \in \operatorname{Hom}\left(\mathbf{Z} / n \mathbf{Z}, T_{n}\right)$ define isomorphic representations iff $\psi=\varphi^{\boldsymbol{g}}$, for some $g \in$ $G$.

It is also useful to consider the faithful, irreducible $\bar{k}$-representations of $\mathbf{Z} / n \mathbf{Z}$, where $\bar{k}$ denotes the algebraic closure of $k$. 
Proposition 2. All irreducible representations of $\mathbf{Z} / n \mathbf{Z}$ over $\bar{k}$ are one-dimensional. Each $\varphi \in \operatorname{Hom}\left(\mathbf{Z} / n \mathbf{Z}, T_{n}\right)$ defines an irreducible representation of dimension 1 , and all these representations are distinct. $G(\bar{k} \mid k)$ acts on the irreducible representations.

Given a $k$-representation $(V, \rho)$, one may form a $\bar{k}$-representation $\left(V \otimes_{k} \bar{k}\right.$, $\rho \otimes$ id), which we write $(\bar{V}, \bar{\rho})$. We describe $(\bar{V}, \bar{\rho})$, where $V=k\left(\zeta_{n}\right)$, and $\rho$ is an injection $\rho: \mathbf{Z} / n \mathbf{Z} \rightarrow T_{n}$. Recall that the Frobenius map $\gamma(x)=x^{2}$ topologically generates $G(\bar{k} \mid k)$, and hence $\gamma$ generates $G(\bar{k} \mid k)$, where $K$ is any finite extension of $k$. Moreover, for $x \in T_{n}, \gamma^{d_{n}}(x)=x$, since $d_{n}=\left[k\left(\zeta_{n}\right): k\right]$. Let $i: k\left(\zeta_{n}\right) \rightarrow \bar{k}$ be any embedding.

Proposition 3. $i \circ \rho$ defines an irreducible $\bar{k}$ representation of $\mathbf{Z} / n \mathbf{Z}$, say $\sigma$. Then $(\bar{V}, \bar{\rho}) \simeq \amalg_{j=0}^{\alpha_{n}-1}\left(\bar{k}, \sigma^{\gamma^{j}}\right)$. This is independent of $i$, since any two choices of $i$ are conjugate under $G(\bar{k} \mid k)$.

Thus, an irreducible $k$-representation amounts to a choice of primitive $n$th roots of unity $\zeta$; the representation $(\bar{V}, \bar{\rho})$ is then defined by

$$
\bar{V}=\bar{k}^{d_{n}}, \bar{\rho}(T)\left(x_{1}, \ldots, x_{d_{n}}\right)=\left(\zeta x_{1}, \zeta^{2} x_{2}, \ldots, \zeta^{2^{\left(d_{n}-1\right)}} x_{d_{n}}\right)
$$

If we let $(V, \rho)$ be a finite-dimensional $k$-representation of a group $G$ we may form the symmetric algebra $k[V]$, and extend the action of $G$ to $k[V] . k[V]^{G}$ will denote the ring of invariants. If $G=\mathbf{Z} / n \mathbf{Z}$, and $\rho$ is a faithful, irreducible representation on $V$,

$$
H^{*}\left(\sum(\mathbf{Z} / n \mathbf{Z} ; \rho) ; k\right) \cong k[V]^{\mathbf{Z} / n \mathbf{Z}} \text {. }
$$

The structure of $k[V]^{\mathbf{Z} / n \mathbf{Z}}$ may be complicated; for our purpose, however, it will suffice to know in what dimensions elements exist. For this purpose, we may tensor up to $\bar{k}$, for

Proposition 4. $k[V]^{G} \otimes_{k} \bar{k} \cong k[V]^{G}$.

Let $(W, \sigma)$ denote any representation of $\mathbf{Z} / n \mathbf{Z}$ over $\bar{k}$. Thus $W \cong \bigoplus_{j=1}^{s} \bar{k}$, and the representation is determined on a generator $T$ of $\mathbf{Z} / n \mathbf{Z}$ by

$$
\rho(T)=\Delta\left(\zeta_{1}, \ldots, \zeta_{s}\right)
$$

where $\Delta\left(\zeta_{1}, \ldots, \zeta_{s}\right)$ denotes the diagonal matrix with entries $\zeta_{1}, \ldots, \zeta_{s}$, nth roots of unity. Choosing a particular primitive $n$th root $\zeta$, we define integers modulo $n \lambda_{i}$ by $\zeta_{i}=\zeta^{\lambda_{i}}$.

Proposition 5. $\bar{k}[V]^{\mathbf{Z} / n \mathbf{Z}} \subseteq k[V]$ may be identified with the subalgebra of $k\left[x_{1}, \ldots, x_{s}\right]$ consisting of all monomials $x_{1}^{e_{1}} \cdots x_{s}^{e_{s}}$ such that

$$
\sum_{j=1}^{s} e_{j} \lambda_{j}=0(\bmod n) \text {. }
$$

Proof. Let $x_{j}$ represent a basis vector for the $j$ th summand of $(V, \rho)$, so $T x_{j}=\zeta_{j} x_{j}=\zeta^{\lambda} x_{j}$ 
It is evident that the action of $T$ respects the monomial basis of $\bar{k}[V]$. Given a monomial $x_{1}^{e_{1}} \cdots x_{s}^{e_{s}}$,

$$
T\left(x_{1}^{e_{1}} \cdots x_{s}^{e_{s}}\right)=\left(\prod_{j=1}^{s} \zeta_{j}^{e_{j}}\right) x_{1}^{e_{1}} \cdots x_{s}^{e_{s}}=\zeta^{\Sigma \lambda_{j} e_{j}} x_{1}^{e_{1}} \cdots x_{s}^{e_{s}},
$$

so $x_{1}^{e_{1}} \cdots x_{s}^{e_{s}} \in \bar{k}[V]^{\mathbf{Z} / n \mathbf{Z}} \Leftrightarrow \sum_{i=1}^{d_{n}} \lambda_{j} e_{j} \equiv 0(\bmod n)$.

Corollary 6. Let $(V, \rho)$ be any faithful, irreducible $\mathbf{Z} / n \mathbf{Z}$-representation over $k$. Then $k[\bar{V}]^{\mathrm{Z} / n \mathrm{Z}}$ may be identified with the subalgebra of $k\left[x_{1}, \ldots, x_{d_{n}}\right]$ consisting of all monomials $x_{1}^{e_{1}} \cdots x_{d_{n}}^{e_{a_{n}}}$ so that $\sum_{i=1}^{d_{n}} 2^{i} e_{i}=0(\bmod n)$.

DEFINITION 7. Define the dyadic expansion number of $n, \nu(n)$, by $\nu(n)=$ $\min _{S}\left\{\sum_{i=1}^{d_{n}} \alpha_{i}\right\}$ where $S=\left\{\left(\alpha_{1}, \ldots, \alpha_{d_{n}}\right) \mid \sum_{i=1}^{d_{n}} \alpha_{i} 2^{i} \equiv 0(\bmod n)\right\}$.

COROllary 8. Let $(V, \rho)$ be as above. Then the first dimension for which $k[V]^{\mathrm{Z} / n \mathrm{Z}}$ is nontrivial is $\nu(n)$.

As remarked before, $k[V]^{\mathbf{Z} / n \mathbf{Z}}$ is endowed with an $\mathbb{Q}(2)$-action. Extending the action $k$-linearly, we obtain an action of $\mathcal{Q}(2)$ on $\bar{k}[\bar{V}]^{\mathbf{Z} / n \mathbf{Z}}$, which satisfies the Cartan formula and the axiom $\mathrm{Sq}^{i}(x)=0$ if $\operatorname{dim} x<i$, but no longer satisfies the condition $\operatorname{Sq}^{n}(x)=x^{2}$ for $\operatorname{dim} x=n$. We wish to describe the $\mathbb{Q}(2)$-action on $k[V]^{\mathrm{Z} / n \mathbf{Z}}$, when this is given in a basis which splits $\bar{V}$ as a direct sum of 1-dimensional representations, as in Corollary 6.

LEMMA 9. In terms of a basis which splits $\bar{V}$ into a sum of one-dimensional representations, say $x_{1}, \ldots, x_{d_{n}}$, the action of $\mathscr{Q}(2)$ on $\bar{k}[\bar{V}]$ is characterized by the Cartan formula, $\bar{k}$-linearity, and the condition $\mathrm{Sq}^{1} x_{j}=x_{j-1}^{2}$. (Here $x_{0}$ is identified with $x_{d_{n}}$.)

Proof. Writing $(\bar{V}, \bar{\rho}) \cong \bigoplus_{j=1}^{d_{n}}\left(\bar{V}_{j}, \bar{\rho}_{j}\right)$, where $\bar{V}_{i} \cong \bar{k}$, and $\rho_{i}(T)(x)=\zeta^{2^{i-1}} x, V$ may be identified with the invariants $\left[\bigoplus_{j=1}^{d_{n}} \bar{V}_{j}\right]^{G(\bar{k} \mid k)}$ where $G(\bar{k} \mid k)$ acts on $\bigoplus_{j=1}^{d_{n}} V_{j}$ by

$$
\left(x_{1}, \ldots, x_{d_{n}}\right)^{\gamma}=\left(x_{d_{n}}^{\gamma}, x_{1}^{\gamma}, \ldots, x_{d_{n}-1}^{\gamma}\right) .
$$

Now, $\mathrm{Sq}^{1} x=x^{2}$ for $x \in V=A_{1}$, so it suffices to check that the formula of the theorem satisfies this condition on $V$. But every element in $\left[\bigoplus_{j=1}^{d_{n}} \bar{V}_{j}\right]^{G(\bar{k} \mid k)}$ is of the form

$$
\left(\alpha, \alpha^{\gamma}, \alpha^{\gamma^{2}}, \ldots, \alpha^{\gamma^{d_{n}-1}}\right), \quad \text { for } \alpha \in k\left(\zeta_{n}\right) .
$$

If $\left(\alpha_{1}, \ldots, \alpha_{d_{n}}\right)=\left(\alpha_{d_{n}}^{\gamma}, \alpha_{1}^{\gamma}, \ldots, \alpha_{d_{n}-1}^{\gamma}\right)$, we obtain $\alpha_{1}=\alpha_{d_{n}}^{\gamma}=\alpha_{d_{n}-1}^{\gamma^{2}}=\cdots=\alpha_{1}^{\gamma^{\alpha_{n}}}$. Thus, $\alpha_{0}$ belongs to the fixed field of $\gamma^{\alpha_{n}}$, which is $k\left(\zeta_{n}\right)$. Applying the formula of the theorem to elements of this type, we obtain

$$
\mathrm{Sq}^{1}\left(\sum_{j=1}^{d_{n}} \alpha^{\gamma^{j}} x_{j+1}\right)=\sum_{j=1}^{d_{n}} \alpha^{\gamma^{j}} x_{j}^{2}=\sum_{j=1}^{d_{n}} \alpha^{\gamma^{j+1}} x_{j+1}^{2}=\left(\sum_{j=1}^{d_{n}} \alpha^{\gamma^{j}} x_{j+1}\right)^{2},
$$

where we interpret $x_{d_{n}+1}$ as $x_{1}$.

3. Systems of parameters in $k[V]^{\mathrm{Z} / n \mathrm{Z}}$. Recall from $\S 1$ the definition of an h.s.o.p. $\left\{\theta_{1}, \ldots, \theta_{k}\right\}$ of a graded $k$-algebra. We say $\left\{\theta_{1}, \ldots, \theta_{k}\right\}$ is $Q(2)$-invariant if the 
ideal $\left(\theta_{1}, \ldots, \theta_{k}\right)$ is preserved by the $\mathcal{Q}(2)$-action, provided the given graded $k$-algebra is an $\mathbb{Q}(2)$-algebra.

Lemma 1. Let $\left\{\theta_{1}, \ldots, \theta_{k}\right\}$ be an h.s.o.p. for a graded algebra $C_{*}$, and $j$ : $C_{*} \rightarrow C_{*}^{\prime}$ a surjective map of graded rings. Then $\left\{j \theta_{1}, \ldots, j \theta_{k}\right\}$ is an h.s.o.p. for $C_{*}^{\prime}$. Similarly, if $C_{*}$ and $C_{*}^{\prime}$ are $\mathcal{Q}(2)$-algebras, and $j$ commutes with the $\mathcal{Q}(2)$-action, then $\left\{j \theta_{1}, \ldots, j \theta_{k}\right\}$ is an $\mathbb{Q}(2)$-invariant h.s.o.p.

Proof. Immediate.

Proposition 2. Suppose $\left\{\theta_{1}, \ldots, \theta_{k}\right\}$ is an h.s.o.p. for $k[V]^{\mathbf{Z} / n \mathbf{Z}}$, and suppose $\operatorname{dim}\left(\theta_{j}\right)=d$ for all $j=1, \ldots, k$. Then $n \mid d$.

Proof. It is clear that $\left\{\theta_{1}, \ldots, \theta_{k}\right\}$ also forms an h.s.o.p. for $\bar{k}[\bar{V}]^{\mathbf{Z} / n \mathbf{Z}}$. Perform a basis change so that $\bar{k}[\bar{V}]$ is identified with the subalgebra of $\bar{k}\left[x_{1}, \ldots, x_{d_{n}}\right]$ defined in Corollary 2.6. Consider the ideal $I \subseteq \bar{k}[\bar{V}]$ generated by all monomials $x_{i} x_{j} ; i \neq j$. Then $\bar{k}[\bar{V}] / I$ has the elements $x_{i}^{s}$ as basis in dimension $s$. The image of $\bar{k}[V]^{\mathbf{Z} / n \mathbf{Z}}$ consists of the subalgebra generated by $x_{1}^{n}, \ldots, x_{d_{n}}^{n}$. In particular, $k[V]^{\mathbf{Z} / n \mathbf{Z}} / I \cap k[V]^{\mathbf{z} / n \mathbf{z}}$ is zero in all dimensions except multiples of $n$, and hence admits h.s.o.p. $\left\{\eta_{1}, \ldots, \eta_{k}\right\}$ with $\operatorname{dim}\left(\eta_{1}\right)=\cdots=\operatorname{dim}\left(\eta_{k}\right)=d$ only for $n \mid d$.

Consider $A_{*}=\bar{k}\left[x_{1}, \ldots, x_{d_{n}}\right]$, with $\mathbf{Z} / n \mathbf{Z}$ acting by $T x_{i}=\zeta^{i^{i-1}} x_{i}, \zeta$ a primitive $n$th root of unity, and $\mathcal{Q}(2)$ acting as in Lemma 2.9.

Lemma 3. The ideal $J$ generated by the elements $\left\{x_{i}+x_{j}\right\}_{1<i, j<d_{n}}$ is $\mathbb{Q}(2)$-invariant. Hence $A_{*} / J$ is an algebra over $\mathbb{Q}(2)$. Moreover, $A_{*} / J \simeq k[x]$, with $\mathrm{Sq}^{1} x=$ $x^{2}$.

Proof. Clear.

Proposition 4. Let $\left\{\theta_{1}, \ldots, \theta_{k}\right\}$ be an $Q(2)$-invariant h.s.o.p. for $A_{*}^{\mathrm{Z} / n \mathbf{Z}}$, with $\operatorname{dim}\left(\theta_{1}\right)=\cdots=\operatorname{dim}\left(\theta_{k}\right)=S$. Then $2^{t+1} \mid S$, where $t$ is the largest integer satisfying $2^{t}<\nu(n)$.

Proof. According to Corollary $2.8, A_{*}^{\mathbf{Z} / n \mathbf{Z}}=0$ for $*<\nu(n)$. Consequently, $B_{*}=0$ also for $*\left\langle\nu(n)\right.$, where $B_{*}=A_{*}^{\mathbf{Z} / n \mathbf{Z}} / J \cap A_{*}^{\mathbf{Z} / n \mathbf{Z}}$. Let $\left\{\eta_{1}, \ldots, \eta_{l}\right\}$ be any $\mathcal{Q}(2)$-invariant h.s.o.p. for $B_{*}, \operatorname{dim}\left(\eta_{1}\right)=\cdots=\operatorname{dim}\left(\eta_{l}\right)=S$. Since $\left\{\eta_{1}, \ldots, \eta_{l}\right\}$ is $Q(2)$-invariant,

$$
\operatorname{Sq}^{i}\left(\eta_{j}\right)=\sum \lambda_{j k}^{i} \eta_{k}
$$

where $\lambda_{j k}^{i} \in B_{i}$. But for $i<\nu(n), B_{i}=0$, so $\mathrm{Sq}^{i}\left(\eta_{j}\right)=0$ for $i<\nu(n)$. Using the decomposability of $\mathrm{Sq}^{i}$ for $i$ not a power of 2, this is equivalent to the requirement $\operatorname{Sq}^{2^{q}}\left(\eta_{j}\right)=0 \forall q \leqslant t$. But, as is well known,

$$
\mathrm{Sq}^{2^{q}}\left(x^{m}\right)=0 \quad \forall q \leqslant t \Rightarrow 2^{t+1} \mid m .
$$

Since a basis for $B_{m}$ is given by $x^{m}$, for $m>\nu(n)$, if the $\eta_{j}$ 's are to form an h.s.o.p., we must have $2^{t+1} \mid s$. Applying the second half of Lemma 1, we see that $\operatorname{dim}\left(\theta_{1}\right)=\cdots=\operatorname{dim}\left(\theta_{k}\right)=S$ is divisible by $2^{t+1}$.

We interpret this geometrically. 
THeOREM 5. Let $\Sigma(\mathbf{Z} / n \mathbf{Z}, \rho)$ act freely on $X \simeq \Pi_{j=1}^{k} S^{m}, X$ finite, and trivially on $H_{*}(X ; k)$, where $\rho$ is an irreducible, faithful representation of $\mathbf{Z} / n \mathbf{Z}, n$ odd. Then

(a) $n \mid(m+1)$,

(b) $2^{t+1} \mid(m+1)$, where $t$ satisfies $2^{t}<\nu(n)<2^{t+1}$.

Proof. By Proposition 1.5 and Theorem 1.7, the $f_{j}$ 's $\left(f_{j}=d_{m+1}\left(z_{j}\right), z_{j}\right.$ the dual to the fundamental class of the $j$ th sphere) form an $Q(2)$-invariant h.s.o.p. for $H^{*}\left(\sum(\mathbf{Z} / n \mathbf{Z}, \rho) ; k\right)$ with $\operatorname{dim}\left(f_{j}\right)=m+1$. Proposition 2 and Proposition 4 now imply the result.

EXAMPLE 6. Let $G=\Sigma(\mathbf{Z} / 31, \rho)$, so $G=\mathbf{Z} / 31 \times{ }_{\rho}(\mathbf{Z} / 2)^{5} . G$ acts freely on $\Pi_{j=1}^{k} S^{m}$, for some $k$, with trivial action on homology only if $8 \mid(m+1)$, since $\nu(31)=5$.

COROLlaRY 7. Let $G$ be any finite group acting freely on $\Pi_{j=1}^{k} S^{2^{q}-1}$, with trivial action on $H_{*}\left(\Pi_{j=1}^{k} S^{2^{q}-1} ; k\right)$. Let $E \subseteq G$ be any elementary abelian 2-subgroup, and $\vartheta \subseteq N_{G}(E)$ an odd order subgroup. Then $\theta$ acts trivially on $E$.

Proof. Apply Theorem 5(a), noting that on $\Pi_{j=1}^{k} S^{2^{a}-1}$, none of the groups $\Sigma(\mathbf{Z} / n \mathbf{Z} ; \rho)$ act freely, and if there were an $\theta \subseteq N_{G}(E)$ which acted nontrivially on $E$, some $\Sigma(\mathbf{Z} / n \mathbf{Z} ; \rho)$ would embed in $G$.

4. The groups $A_{4}$ and $\Sigma(Z / 7 Z ; \rho)$. In this section, we will prove that the groups $A_{4}=\Sigma(\mathbf{Z} / 3 \mathbf{Z} ; \rho)$ and $\Sigma(\mathbf{Z} / 7 \mathbf{Z} ; \rho)$ do not act freely on $\Pi_{j=1}^{k} S^{n}$ for any values of $k$ and $n$, where as usual $\rho$ denotes an irreducible, faithful representation of the odd order cyclic group in question. Using the terminology of $\$ 2$, we observe that $\nu(3)=2$, and $\nu(7)=3$. Consequently $H^{1}\left(A_{4} ; k\right)=H^{1}(\Sigma(\mathbf{Z} / 7 \mathbf{Z}, \rho) ; k)=$ $H^{2}(\Sigma(\mathbf{Z} / 7 \mathbf{Z} ; \rho) ; k)=0$. Let $A_{*}=\bar{k}\left[x_{1}, x_{2}\right]$ for $A_{4}$, and $A_{*}=\bar{k}\left[x_{1}, x_{2}, x_{3}\right]$ for $\Sigma(\mathbf{Z} / 7 \mathbf{Z}, \rho)$. Let $B_{*}=A_{*}^{\mathbf{Z} / 3 \mathbf{Z}}$ and $B_{*}=A_{*}^{\mathbf{Z} / 7 \mathbf{Z}}$ respectively.

Proposition 1. Let $\left\{\theta_{1}, \ldots, \theta_{t}\right\}$ be a collection of homogeneous elements in $H^{*}(G ; k)$, where $G=A_{4}$ or $\Sigma(\mathbf{Z} / 7 \mathbf{Z}, \rho)$, so that $\operatorname{dim}\left(\theta_{1}\right)=\cdots=\operatorname{dim}\left(\theta_{t}\right)=2 d$. Suppose the ideal $\left(\theta_{1}, \ldots, \theta_{t}\right)$ is $\mathbb{Q}(2)$-invariant. Then $\theta_{i}$ is a square in $A_{*}$ for all $i$.

Proof. By the above remarks, since $H^{*}\left(A_{4} ; k\right)=0$ for $*=1$, and $H^{*}(\Sigma(\mathbf{Z} / 7 \mathbf{Z}, \rho) ; k)=0$ for $*=1,2$, if $\left(\theta_{1}, \ldots, \theta_{t}\right)$ is $Q(2)$-invariant, we must have $\operatorname{Sq}^{1}\left(\theta_{i}\right)=0$ in the case of $A_{4}$, and $\operatorname{Sq}^{1}\left(\theta_{i}\right)=\operatorname{Sq}^{2}\left(\theta_{i}\right)=0$ in the case of $\Sigma(\mathbf{Z} / 7 \mathbf{Z}, \rho)$. Since $\operatorname{dim}\left(\theta_{i}\right)=2 d, Q_{0}$ vanishes on $\theta_{i}$ by Proposition $1.2(\mathrm{c})$. In the case of $\Sigma(\mathrm{Z} / 7 \mathrm{Z}, \rho), Q_{2}=\left[\mathrm{Sq}^{1}, \mathrm{Sq}^{2}\right]$ vanishes on $\theta_{i}$ as well. $H^{*}\left(A_{4} ; k\right)$ is a subalgebra of $H^{*}(\mathbf{Z} / 2 \mathbf{Z} \times \mathbf{Z} / 2 \mathbf{Z}, k)$, consequently $Q_{j}$ may be expressed as a linear combination of $Q_{0}$ and $Q_{1}$ for all $j$. Hence $Q_{j} \theta_{i}=0 \forall j$, which by Corollary 1.4 implies that $\theta_{i}$ is a square. Similarly, $\left[k\left(\zeta_{7}\right): k\right]=3$, so on $H^{*}(\Sigma(\mathbf{Z} / 7 \mathbf{Z}, \rho)), Q_{j}$ may be expressed as a linear combination of $Q_{0}, Q_{1}$, and $Q_{2}$, implying $Q_{j} \theta_{i}=0 \forall_{i}$, so $\theta_{i}$ is a square in $A_{*}$.

Proposition 2. Let $\theta \in B_{*}$ be a homogeneous element in $B_{*}$, with $\theta=\eta^{2}$ for some $\eta \in A_{*}$. Then $\eta \in B_{*}$. 
Proof. The map $x \rightarrow x^{2}$ from $A_{S}$ to $A_{2 S}$ is $k$-linear and an injection. Hence $\eta$ is uniquely determined. If $\theta \in B_{2 S}$, then $\theta$ is a sum $\sum a_{\left(e_{1}, \ldots, e_{l}\right)} x_{1}^{e_{1}} \cdots x_{l}^{e_{l}}$, where the $l$-tuples $\left(e_{1}, \ldots, e_{l}\right)$ range over all solutions to the equation $\sum 2^{i-1} e_{i} \equiv 0(\bmod n)$, and $n=3$ or $7, l=2$ or $3 . \theta$ is a square in $A_{*}$ if and only if each $e_{j}$ is even, say $e_{j}=2 f_{j}$. Thus, $\eta=\sum \sqrt{a_{\left(e_{1}, \ldots, e_{l}\right)}} x_{1}^{f_{1}} \cdots x_{l}^{f_{l}}$. Now, $\sum 2^{i-1} f_{i}=\frac{1}{2} \sum 2^{i-1} e_{i} \equiv 0$ $(\bmod n)$, since $n$ is odd.

Let $A_{*}^{2}$ and $B_{*}^{2}$ denote the subalgebras of squares in $A_{*}$ and $B_{*}$. The above proposition shows that $A_{*}^{2} \cap B_{*}=B_{*}^{2}$.

Proposition 3. Let $\theta_{1}, \ldots, \theta_{s} \in B_{*}^{2}, \operatorname{dim}\left(\theta_{1}\right)=\cdots=\operatorname{dim}\left(\theta_{s}\right)$, and suppose that $\xi=\sum \lambda_{i} \theta_{i}$, where $\xi \in B_{*}^{2}$ is homogeneous, and the $\lambda_{i}$ 's are homogeneous elements of $A_{*}$. Then there is an expression $\xi=\sum \lambda_{i}^{\prime} \theta_{i}$, with $\lambda_{i}^{\prime}$ homogeneous, $\lambda_{i}^{\prime} \in B_{*}^{2}$.

Proof. We first point out that $A_{*}=\bar{k}\left[x_{1}, \ldots, x_{l}\right]$ is free as an $A_{*}^{2}$-module, with basis consisting of all monomials $x_{1}^{d_{1}} \cdots x_{l}^{d_{l}}$, with $d_{j}=0$ or 1 . Given a monomial $\mu$ in the above basis, let $\pi_{\mu}$ denote the projection on the $\mu$ th factor in the $A_{*}^{2}$ module $A_{*} \cdot \pi_{\mu}$ is $A_{*}^{2}$-linear. If we have an expression $\xi=\Sigma \lambda_{i} \theta_{i}$, as in the theorem, we apply $\pi_{1}$ to the expression to obtain $\pi_{1}(\xi)=\Sigma \pi_{1}\left(\lambda_{i}\right) \theta_{i}$. Since $\xi$ is a square, $\pi_{1}(\xi)=\xi$, and $\pi_{1}\left(\lambda_{i}\right) \in A_{*}^{2}$, so we may suppose that the $\lambda_{i}$ 's are squares.

Let $\left\{\chi_{i}\right\}$ be a complete set of irreducible characters of $\mathbf{Z} / n \mathbf{Z}$ over $\bar{k}$. Let $p_{i}$ denote the projection on the $\chi_{i}$-isotypical component (see [6]). The $p_{i}$ 's are $B_{*}$-linear, and moreover $p_{i}\left(A_{*}^{2}\right) \subseteq A_{*}^{2}$. If $\xi=\sum \lambda_{i} \theta_{i}$, with $\xi, \theta_{1}, \ldots, \theta_{s} \in B_{*}^{2}$, and $\lambda_{i} \in$ $A_{*}^{2}$, by applying $p_{0}$, if $\chi_{0}$ is the trivial character, we obtain $p_{0}(\xi)=\Sigma p_{0}\left(\lambda_{i}\right) \theta_{i}$, and $p_{0}(\xi)=\xi$, since $\xi \in B_{*}$, so $\xi=\sum p_{0}\left(\lambda_{i}\right) \theta_{i}$. Setting $\lambda_{i}^{\prime}=p_{0}\left(\lambda_{i}\right)$, we obtain the theorem, since $\lambda_{i}^{\prime} \in A_{*}^{2} \cap B_{*}=B_{*}^{2}$, by Proposition 2 .

THEOREM 4. There does not exist a free $G$-action on $\Pi_{i=1}^{k} S^{n}$, with trivial action on homology, for $G=A_{4}$ or $\Sigma(\mathbf{Z} / 7 \mathbf{Z}, \rho)$.

Proof. According to Proposition 1.5 and Theorem 1.7, it suffices to prove that $H^{*}(G ; k)$ admits no $Q(2)$-invariant system of parameters $\left\{\theta_{1}, \ldots, \theta_{s}\right\}$ with $\operatorname{dim}\left(\theta_{1}\right)$ $=\operatorname{dim}\left(\theta_{2}\right)=\cdots=\operatorname{dim}\left(\theta_{s}\right)=n+1$. Proposition 1 and Proposition 2 guarantee that each $\theta_{i}$ is a square in $B_{*}$, say $\theta_{i}=\zeta_{i}$. We claim that the ideal $\left(\zeta_{1}, \ldots, \zeta_{s}\right)$ is $\mathcal{Q}(2)$-invariant. Let $\operatorname{Sq}^{j}\left(\theta_{i}\right)=\sum \lambda_{i k}^{j} \theta_{k}$. Since the $\theta$ 's are squares, $\operatorname{Sq}^{2 j}\left(\theta_{i}\right)=\left(\operatorname{Sq}^{j}\left(\zeta_{i}\right)\right)^{2}$, and $\operatorname{Sq}^{2 j+1}\left(\theta_{i}\right)=0$. To obtain an expression for $\operatorname{Sq}^{j}\left(\zeta_{i}\right)$ in terms of $\zeta_{1}, \ldots, \zeta_{s}$, we write $\left(\mathrm{Sq}^{j}\left(\zeta_{i}\right)\right)^{2}=\sum \lambda_{i k}^{2 j} \theta_{k}=\sum \lambda_{i k}^{2 j} \zeta_{k}^{2}$. But Proposition 3 gives that $\lambda_{i k}^{2 j}$ may be chosen to be a square, say $\lambda_{i k}^{2 j}=\left(\mu_{i k}^{j}\right)^{2}$, so $\left(\mathrm{Sq}^{j}\left(\zeta_{i}\right)\right)^{2}=\Sigma\left(\mu_{i k}^{j}\right)^{2 \zeta_{k}^{2}}$, or $\mathrm{Sq}^{j}\left(\zeta_{i}\right)=\Sigma \mu_{i k}^{j} \zeta_{k}$, which was to be shown. Moreover, $\left\{\zeta_{1}, \ldots, \zeta_{s}\right\}$ is an h.s.o.p. for $H^{*}(G ; k)$, since $\left\{\zeta_{1}^{2}, \ldots, \zeta_{s}^{2}\right\}=\left\{\theta_{1}, \ldots, \theta_{s}\right\}$ is. Consequently, if $\operatorname{dim}\left(\theta_{1}\right)=\cdots=\operatorname{dim}\left(\theta_{s}\right)=2^{m} l$, where $l$ is odd, we have demonstrated the existence of an $\mathcal{Q}(2)$-invariant h.s.o.p. $\left\{\zeta_{1}, \ldots, \theta_{s}\right\}$, with $\operatorname{dim}\left(\theta_{i}\right)=2^{m-1} l$. Inductively, we may produce an h.s.o.p. $\left\{\theta_{1}, \ldots, \theta_{s}\right\}$ with $\operatorname{dim}\left(\theta_{i}\right)=l$, where $l$ is odd. We claim, however, that this is impossible. Let $J$ be as in Lemma 3.3. Then $B_{*} / J \cap B_{*}$ is a graded $Q$ (2)-invariant subalgebra of $k[x]$, and $B_{*} / J \cap B_{*}=0$ for $*=1$. Consequently, $B_{*} / J \cap B_{*}$ admits no $Q(2)$-invariant h.s.o.p. in odd dimensions, for $\mathrm{Sq}^{1}$ is nonzero on $x^{2 i+1}$. By Lemma 3.1, $B_{*}$ admits no $\mathbb{Q}(2)$-invariant h.s.o.p. in odd dimensions, which gives a contradiction. 


\section{REFERENCES}

1. J. F. Adams and C. W. Wilkerson, Finite $H$-spaces and algebras over the Steenrod algebra, Ann. of Math. 111 (1980), 95-143.

2. G. Carlsson, On the non-existence of free actions of elementary abelian groups on products of spheres, Amer. J. Math. 102 (1980), 1147-1157.

3. P. Conner, On the action of a finite group on $S^{n} \times S^{n}$, Ann. of Math. (2) 66 (1957), 586-588.

4. J. Milnor, Groups which act on $S^{n}$ without fixed points, Amer. J. Math. 79 (1957), 623-630.

5. R. Oliver, Proc. Aarhus Sympos. on Algebraic Topology, 1978, Lecture Notes in Math., vol. 763, Springer-Verlag, Berlin and New York.

6. R. P. Stanley, Invariants of finite groups and their applications to combinatorics, Bull. Amer. Math. Soc. (N.S.) 1 (1979), 475-511.

7. E. Stein, Free actions on products of spheres, Michigan Math. J. (to appear).

8. L. Dornhoff, Group representation theory, Dekker, New York, 1971-1972.

Department of Mathematics, University of California-San Diego, La Jolla, California 92093 